Supplement of Arch. Anim. Breed., 62, 393-402, 2019

https://doi.org/10.5194/aab-62-393-2019-supplement

(C) Author(s) 2019. This work is distributed under

the Creative Commons Attribution 4.0 License.

(c) (i)

Supplement of

\title{
Differentiation of some Pramenka sheep breeds based on morphometric characteristics
}

\section{Božidarka Marković et al.}

Correspondence to: Božidarka Marković (bozidarkam@ucg.ac.me)

The copyright of individual parts of the supplement might differ from the CC BY 4.0 License. 


\section{Supplement Tables:}

Table 3a: Pearson correlation coefficients of morphometric traits of Istrian Pramenka (below diagonal) and Bela Krajina

5 Pramenka (above diagonal)

\begin{tabular}{|c|c|c|c|c|c|c|c|c|c|}
\hline \multicolumn{10}{|c|}{$\begin{array}{c}\text { Pearson Correlation Coefficients, I = } 64 \text { BK } 64 \\
\text { Prob }>|\mathbf{r}| \text { under H0: Rho=0 }\end{array}$} \\
\hline & WH & RH & BL & CD & CW & RW & $\mathrm{CC}$ & $\mathrm{CBC}$ & BW \\
\hline \multirow{2}{*}{ WH } & \multirow{2}{*}{1} & 0,804 & 0,382 & 0,452 & 0,280 & 0,279 & 0,352 & 0,376 & 0,345 \\
\hline & & $<.0001$ & 0,000 & $<.0001$ & 0,009 & 0,009 & 0,001 & 0,000 & 0,001 \\
\hline \multirow{2}{*}{ RH } & 0,791 & \multirow{2}{*}{1} & 0,385 & 0,502 & 0,319 & 0,290 & 0,412 & 0,445 & 0,481 \\
\hline & $<.0001$ & & 0,000 & $<.0001$ & 0,003 & 0,007 & $<.0001$ & $<.0001$ & $<.0001$ \\
\hline \multirow{2}{*}{ BL } & 0,425 & 0,345 & \multirow{2}{*}{1} & 0,462 & 0,364 & 0,263 & 0,398 & 0,486 & 0,507 \\
\hline & 0,001 & 0,005 & & $<.0001$ & 0,001 & 0,014 & 0,000 & $<.0001$ & $<.0001$ \\
\hline \multirow{2}{*}{ CD } & 0,402 & 0,486 & 0,338 & \multirow{2}{*}{1} & 0,580 & 0,406 & 0,687 & 0,486 & 0,769 \\
\hline & 0,001 & $<.0001$ & 0,006 & & $<.0001$ & 0,000 & $<.0001$ & $<.0001$ & $<.0001$ \\
\hline \multirow{2}{*}{ CW } & 0,177 & 0,239 & 0,220 & 0,412 & \multirow{2}{*}{1} & 0,523 & 0,714 & 0,579 & 0,731 \\
\hline & 0,161 & 0,058 & 0,080 & 0,001 & & $<.0001$ & $<.0001$ & $<.0001$ & $<.0001$ \\
\hline \multirow{2}{*}{ RW } & 0,375 & 0,434 & 0,432 & 0,449 & 0,547 & \multirow{2}{*}{1} & 0,342 & 0,416 & 0,466 \\
\hline & 0,002 & 0,000 & 0,000 & 0,000 & $<.0001$ & & 0,001 & $<.0001$ & $<.0001$ \\
\hline \multirow{2}{*}{$\mathrm{CC}$} & 0,451 & 0,522 & 0,277 & 0,685 & 0,575 & 0,658 & \multirow{2}{*}{1} & 0,464 & 0,790 \\
\hline & 0,000 & $<.0001$ & 0,027 & $<.0001$ & $<.0001$ & $<.0001$ & & $<.0001$ & $<.0001$ \\
\hline \multirow{2}{*}{$\mathrm{CBC}$} & 0,210 & 0,292 & 0,355 & 0,536 & 0,470 & 0,522 & 0,542 & \multirow{2}{*}{1} & 0,555 \\
\hline & 0,096 & 0,019 & 0,004 & $<.0001$ & $<.0001$ & $<.0001$ & $<.0001$ & & $<.0001$ \\
\hline \multirow{2}{*}{ BW } & 0,438 & 0,491 & 0,418 & 0,737 & 0,667 & 0,695 & 0,839 & 0,745 & 1 \\
\hline & 0,000 & $<.0001$ & 0,001 & $<.0001$ & $<.0001$ & $<.0001$ & $<.0001$ & $<.0001$ & \\
\hline
\end{tabular}

WH - wither height, RH - rump height, BL - body length, CD - chest depth, CW - chest width, RW - rump width, $\mathrm{CC}$ - chest circumference, $\mathrm{CBC}$ - cannon bone circumference, $\mathrm{BW}$ - body weight. 
Table 3b: Pearson correlation coefficients of morphometric traits of Pivska Pramenka (below diagonal) and Sjenicka sheep (above diagonal)

\begin{tabular}{|c|c|c|c|c|c|c|c|c|c|}
\hline \multicolumn{10}{|c|}{$\begin{array}{c}\text { Pearson Correlation Coefficients, } P=90 \mathrm{~S}=59 \\
\text { Prob }>|\mathbf{r}| \text { under H0: } \text { Rho }=0\end{array}$} \\
\hline & WH & RH & BL & CD & CW & RW & $\mathrm{CC}$ & $\mathrm{CBC}$ & BW \\
\hline \multirow{2}{*}{ WH } & \multirow{2}{*}{1} & 0,926 & 0,516 & 0,494 & 0,203 & 0,180 & 0,406 & 0,497 & 0,491 \\
\hline & & $<.0001$ & $<.0001$ & $<.0001$ & 0,122 & 0,172 & 0,001 & $<.0001$ & $<.0001$ \\
\hline \multirow{2}{*}{ RH } & 0,667 & \multirow{2}{*}{1} & 0,549 & 0,424 & 0,269 & 0,225 & 0,329 & 0,399 & 0,435 \\
\hline & $<.0001$ & & $<.0001$ & 0,001 & 0,039 & 0,086 & 0,011 & 0,002 & 0,001 \\
\hline \multirow{2}{*}{ BL } & 0,331 & 0,475 & \multirow{2}{*}{1} & 0,380 & 0,461 & 0,403 & 0,333 & 0,398 & 0,556 \\
\hline & 0,001 & $<.0001$ & & 0,003 & 0,000 & 0,002 & 0,010 & 0,002 & $<.0001$ \\
\hline \multirow{2}{*}{ CD } & 0,189 & 0,117 & 0,016 & \multirow{2}{*}{1} & 0,405 & 0,509 & 0,814 & 0,594 & 0,818 \\
\hline & 0,074 & 0,273 & 0,882 & & 0,002 & $<.0001$ & $<.0001$ & $<.0001$ & $<.0001$ \\
\hline \multirow{2}{*}{$\mathrm{CW}$} & 0,014 & 0,135 & 0,182 & 0,186 & \multirow{2}{*}{1} & 0,741 & 0,523 & 0,108 & 0,583 \\
\hline & 0,894 & 0,205 & 0,086 & 0,079 & & $<.0001$ & $<.0001$ & 0,414 & $<.0001$ \\
\hline \multirow{2}{*}{ RW } & $-0,082$ & 0,029 & 0,066 & 0,224 & 0,620 & \multirow{2}{*}{1} & 0,559 & 0,191 & 0,602 \\
\hline & 0,440 & 0,790 & 0,534 & 0,034 & $<.0001$ & & $<.0001$ & 0,146 & $<.0001$ \\
\hline \multirow{2}{*}{$\mathrm{CC}$} & $-0,048$ & $-0,082$ & 0,014 & 0,081 & 0,276 & $-0,014$ & \multirow{2}{*}{1} & 0,491 & 0,968 \\
\hline & 0,655 & 0,443 & 0,895 & 0,450 & 0,009 & 0,896 & & $<.0001$ & $<.0001$ \\
\hline \multirow{2}{*}{$\mathrm{CBC}$} & 0,344 & 0,321 & 0,371 & $-0,156$ & 0,001 & $-0,171$ & 0,216 & \multirow{2}{*}{1} & 0,530 \\
\hline & 0,001 & 0,002 & 0,000 & 0,143 & 0,995 & 0,108 & 0,041 & & $<.0001$ \\
\hline \multirow{2}{*}{ BW } & 0,305 & 0,338 & 0,460 & 0,056 & 0,108 & $-0,339$ & 0,454 & 0,457 & \multirow{2}{*}{1} \\
\hline & 0,004 & 0,001 & $<.0001$ & 0,600 & 0,310 & 0,001 & $<.0001$ & $<.0001$ & \\
\hline
\end{tabular}

WH - wither height, $\mathrm{RH}$ - rump height, $\mathrm{BL}$ - body length, $\mathrm{CD}$ - chest depth, $\mathrm{CW}$ - chest width, RW - rump width, $\mathrm{CC}$ -

10 chest circumference, $\mathrm{CBC}$ - cannon bone circumference, BW - body weight. 
Table 3c: Pearson correlation coefficients of morphometric traits of Bardoka (below diagonal) and Žuja (above diagonal)

\begin{tabular}{|c|c|c|c|c|c|c|c|c|c|}
\hline \multicolumn{10}{|c|}{$\begin{array}{c}\text { Pearson Correlation Coefficients, } B=44, Z=38 \\
\text { Prob }>|r| \text { under H0: } \text { Rho }=0\end{array}$} \\
\hline & WH & RH & BL & CD & $\mathrm{CW}$ & RW & $\mathrm{CC}$ & CBC & BW \\
\hline \multirow{2}{*}{ WH } & \multirow{2}{*}{1} & 0,936 & 0,538 & 0,648 & 0,444 & 0,147 & 0,433 & 0,403 & 0,627 \\
\hline & & $<.0001$ & 0,001 & $<.0001$ & 0,005 & 0,378 & 0,007 & 0,012 & $<.0001$ \\
\hline \multirow{2}{*}{ RH } & 0,895 & \multirow{2}{*}{1} & 0,578 & 0,529 & 0,363 & 0,050 & 0,508 & 0,404 & 0,672 \\
\hline & $<.0001$ & & 0,000 & 0,001 & 0,025 & 0,764 & 0,001 & 0,012 & $<.0001$ \\
\hline \multirow{2}{*}{ BL } & 0,402 & 0,359 & \multirow{2}{*}{1} & 0,710 & 0,495 & 0,029 & 0,498 & 0,656 & 0,768 \\
\hline & 0,007 & 0,017 & & $<.0001$ & 0,002 & 0,861 & 0,002 & $<.0001$ & $<.0001$ \\
\hline \multirow{2}{*}{ CD } & 0,594 & 0,755 & 0,217 & \multirow{2}{*}{1} & 0,499 & 0,234 & 0,547 & 0,622 & 0,731 \\
\hline & $<.0001$ & $<.0001$ & 0,157 & & 0,001 & 0,157 & 0,000 & $<.0001$ & $<.0001$ \\
\hline \multirow{2}{*}{ CW } & $-0,263$ & $-0,206$ & $-0,196$ & $-0,160$ & \multirow{2}{*}{1} & $-0,037$ & 0,512 & 0,583 & 0,632 \\
\hline & 0,084 & 0,180 & 0,203 & 0,299 & & 0,827 & 0,001 & 0,000 & $<.0001$ \\
\hline \multirow{2}{*}{ RW } & 0,159 & 0,178 & 0,031 & 0,112 & 0,357 & \multirow{2}{*}{1} & 0,010 & 0,108 & $-0,048$ \\
\hline & 0,304 & 0,248 & 0,844 & 0,468 & 0,017 & & 0,954 & 0,518 & 0,774 \\
\hline \multirow{2}{*}{$\mathrm{CC}$} & 0,248 & 0,264 & $-0,006$ & 0,448 & 0,316 & 0,246 & \multirow{2}{*}{1} & 0,463 & 0,740 \\
\hline & 0,105 & 0,084 & 0,972 & 0,002 & 0,037 & 0,108 & & 0,003 & $<.0001$ \\
\hline \multirow{2}{*}{ CBC } & 0,505 & 0,545 & 0,389 & 0,469 & $-0,171$ & $-0,049$ & 0,323 & \multirow{2}{*}{1} & 0,714 \\
\hline & 0,001 & 0,000 & 0,009 & 0,001 & 0,268 & 0,753 & 0,032 & & $<.0001$ \\
\hline \multirow{2}{*}{ BW } & 0,388 & 0,387 & 0,398 & 0,502 & 0,209 & 0,239 & 0,914 & 0,450 & \multirow[t]{2}{*}{1} \\
\hline & 0,009 & 0,010 & 0,008 & 0,001 & 0,174 & 0,118 & $<.0001$ & 0,002 & \\
\hline
\end{tabular}

WH - wither height, RH - rump height, BL - body length, CD - chest depth, CW - chest width, RW - rump width, CC chest circumference, $\mathrm{CBC}$ - cannon bone circumference, BW - body weight. 
